\title{
Pancreatic Pseudotumour in Seronegative Autoimmune Pancreatitis: A Rare case in the Caribbean
}

\section{Elizabeth Guelmo-Daisley*, Shiva Sarwan, Ariadne Saunders and Osei Fraser}

Department of Surgery, Port-Of-Spain General Hospital, Trinidad

*Corresponding Author: Elizabeth Guelmo-Daisley, Department of Surgery, Port-Of-Spain General Hospital, Trinidad.
Received: December 01, 2021

Published: January 17, 2022

(C) All rights are reserved by Elizabeth

Guelmo-Daisley., et al.

\begin{abstract}
Background: Autoimmune pancreatitis (AIP) is commonly found in Asia and Japan but its incidence in most of the western world is yet to be determined. There are two histologically distinct forms of the disease: type 1 and type 2 AIP. AIP may appear clinically and radiologically similar to pancreatic adenocarcinoma, but with fundamentally different treatment strategies.

Case Presentation: A non-alcoholic 42-year-old female with no medical issues presented with painless and progressive clinical and biochemical obstructive jaundice. Initial focused hepatobiliary ultrasound excluded cholelithiasis, and CECT and MRI abdomen with contrast/MRCP imaging of the biliary tree was highly suggestive of a pancreatic head neoplasm. Her Ca 19.9 levels were elevated and her IgG4 serology was normal. A pancreaticoduodenectomy was done. Histological examination of the specimen revealed nil evidence of pancreatic adenocarcinoma, but a robust lymphoplasmacytic inflammatory infiltrate with storiform fibrosis in keeping with autoimmune pancreatitis.
\end{abstract}

Discussion: Despite the existence of and adherence to robust AIP international guidelines, pancreatic pseudotumours represent a distinct clinical entity that is difficult to distinguish from pancreatic adenocarcinoma based on clinical, radiological and serological investigations. Thus, the incidence of patients having unnecessary surgery for AIP due to misdiagnosis remains around 9.9\%, with the inherent associated morbidity [1].

Keywords: Autoimmune Pancreatitis; Biliary Tree; Pancreatic Adenocarcinoma

\section{Introduction}

Autoimmune pancreatitis (AIP) was first described by Sarles., et al. in 1961, as a chronic sclerosing pancreatitis. The term AIP was coined by Yoshida., et al. in 1995 [2]. While the incidence of AIP is highest in Asia and Japan, there are studies that suggest the incidence of this pathological process is on the rise in Europe and North America [1]. The incidence of AIP in Japan was found to be 3.1 per 100000 persons with a prevalence of approximately 10.1 per 100000 persons. The male to female sex ratio was discovered to be 2.94 with the mean age at diagnosis being 64.8 years [3].

The term AIP is dichotomous in nature, referring to two distinct entities: type 1 and type 2 AIP. Infiltration of the tissues with IgG4- positive plasma cells with storiform fibrosis and obliterative phlebitis is characteristic of type 1 AIP. Type 1 AIP is also referred to as lymphoplasmacytic sclerosing pancreatitis (LPSP). Type 2 AIP is characterized by the presence of granulocytic epithelial lesions and is also referred to as idiopathic duct- centric pancreatitis (IDCP). Elevated IgG titres of $\geq 1800 \mathrm{mg} / \mathrm{dl}$ and elevated IgG 4 levels of $\geq 140$ $\mathrm{mg} / \mathrm{dl}$ are associated with type 1 AIP. However, type 2 AIP is not associated with elevated IgG levels. Pancreatic adenocarcinoma may also be associated with mild elevations in IgG4 levels which may contribute to diagnostic dilemma.

Type 1 AIP is three times as common in males than in females with the average age of onset being 60 . There is no clear etiological 
reason for this gender difference. Whereas there is equal gender predilection with type 2 AIP and a younger age of onset (on average 43 years). Type 1 AIP typically presents with painless obstructive jaundice [2]. An international multicentre survey of AIP in 15 institutions from 8 countries conducted by Kamisawa., et al. revealed that up to $75 \%$ of patients with type 1 AIP presented with obstructive jaundice [3]. Whereas patients with Type 2 AIP typically present with recurrent bouts of acute pancreatitis. Type 1 AIP may also be associated with exocrine and endocrine dysfunction and up to $60 \%$ of persons have extra pancreatic manifestations of the disease [2].

\section{Case Presentation}

A 42-year-old female presented to hospital with a three- day history of nausea, vomiting, and mild epigastric discomfort with a two-week history of having pale stools, dark urine and icterus. There was no history of pruritus. There were no symptoms of anemia and no history of weight loss. Nil other significant history was noted. The patient's blood investigations revealed an obstructive jaundice with elevated liver enzymes. Her total bilirubin was 2.4 $\mathrm{mg} / \mathrm{dl}$ with a direct bilirubin level of $1.9 \mathrm{mg} / \mathrm{dl}$. Her amylase levels were normal, but lipase levels were more than three times the upper limit. Ca 19-9 levels were found to be mildly elevated as well (112.5 U/ml, normal range: 0-27 U/ml) and IgG4 levels were normal (12.8 mg/dl, normal range: 4-86 mg/dL).

An abdominal ultrasound noted that the pancreatic head was diffusely enlarged and hypoechoic with ill- defined borders. The pancreatic head and body measured $4.1 \mathrm{~cm}$ and $2.5 \mathrm{~cm}$ in thickness respectively, with the common bile duct (CBD) measured 1.35 $\mathrm{cm}$. The gallbladder was also distended measuring $12 \mathrm{~cm}$ in length. There was no evidence of wall thickening or pericholecystic fluid or calculi. A CT scan of the abdomen with pancreatic protocol revealed focal enlargement suspicious for a mass lesion in pancreatic head with some peri- pancreatic oedema. There was no pancreatic duct dilation but there was prominence of the CBD and the common hepatic duct (CHD). There was nil dilation of the intra- hepatic ducts and nil biliary calculi. While these findings were suggestive of an early pancreatitis, malignancy could not be definitively ruled out.

The patient's symptoms and biochemical jaundice settled with conservative management, and she was discharged with plans for an urgent outpatient MRI. MRI is not readily available in all our public institutions. Two weeks later whilst pending MRI, the patient's obstructive jaundice recurred with a marked increase in the direct bilirubin to 9. A repeat CT abdomen was done which demonstrated a similar pattern of findings but with new CBD and CHD dilatation measuring $1.6 \mathrm{~cm}$ in diameter each with intra hepatic duct dilatation. The main pancreatic duct [MPD] was normal in calibre and there were a few enlarged peri- pancreatic lymph nodes. CT chest excluded any pulmonary metastatic lesions.

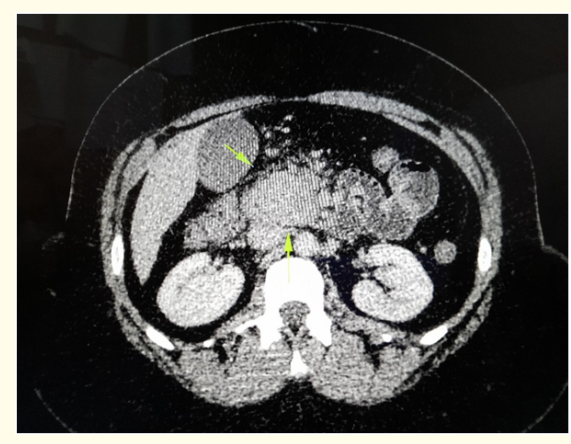

Figure 1: CT scan showing pancreatic head mass. Green arrows directed at lesion.

MRCP and contrast enhanced MRI of the abdomen with Gadolinium, done 1 month after the onset of her symptoms, confirmed a pancreatic head mass measuring $3.9 \times 4.5 \times 6.2 \mathrm{~cm}$ in size, abutting the superior mesenteric vein, the left renal vein and the inferior vena cava. There was no evidence of vascular invasion or thrombus and no lymphadenopathy. There were clear fascial planes with proximal biliary tree dilatation, with a CBD of $1.6 \mathrm{~cm}$ and a prominent MPD. The overall features were highly suggestive of a pancreatic neoplasm.

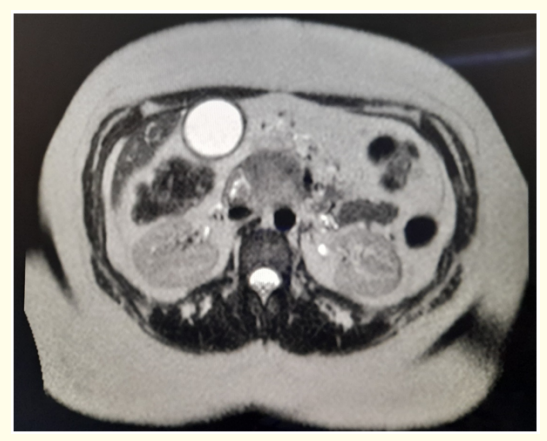

Figure 2: MRI image highlighting pancreatic head mass and distended gallbladder. 
After extensive counselling, the patient underwent a Whipple's procedure (pancreaticoduodenectomy). At laparotomy, a 4 x $5 \mathrm{~cm}$ mass occupying the majority of the pancreatic head was noted with clear fascial planes. The procedure was uneventful, with HDU admission for 5 days, and subsequent hospital discharge at 21 days post op. Histopathological examination of the specimen excluded a pancreatic malignancy with eight benign lymph nodes. A robust lymphoplasmacytic inflammatory infiltrate in the form of lymphoid follicles and sheets of periductal plasma cells and focal storiform fibrosis with associated eosinophils was found. These features were in keeping with a IgG4 related autoimmune pancreatitis. One year post surgery, the patient remains clinically well with no complications.

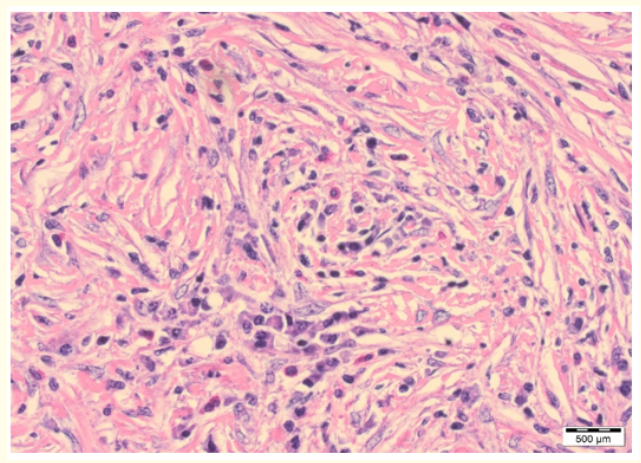

Figure 3: Histology slide showing plasma cells and oeosinophils with storiform fibrosis.

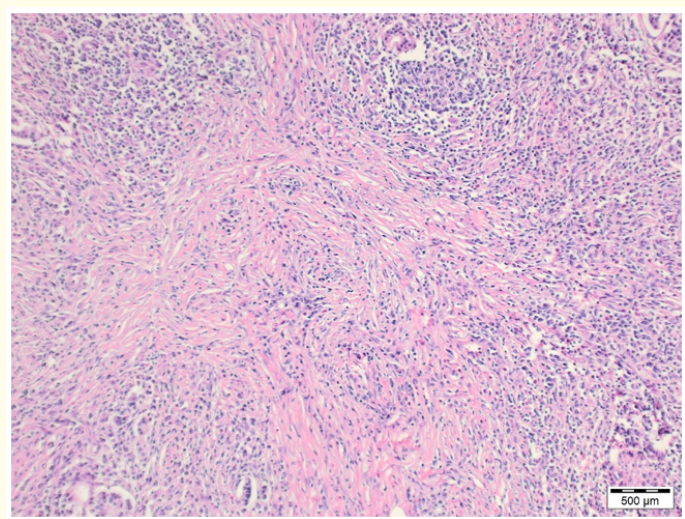

Figure 4: Histology slide showing storiform fibrosis within low power field.

\section{Discussion}

The distinction between pancreatic adenocarcinoma and autoimmune pancreatitis is a difficult one to make. In fact, in one study conducted by de Castro., et al. at the Academic Medical Centre in Amsterdam in the Netherlands, it was found that of the 639 patients undergoing a pancreaticoduodenectomy or Whipple's procedure for presumably pancreatic adenocarcinoma, 63 of these patients were found to have either type 1 AIP or chronic focal pancreatitis [1].

Due to the tremendous difference in the management and prognosis of pancreatic adenocarcinoma versus AIP, all attempts to rule out pancreatic adenocarcinoma must be done. This may not always be possible, however. There are several different diagnostic guidelines that may be employed to make the definitive diagnosis of AIP.

These include:

- $\quad$ The Japanese Pancreas Society Guidelines (2002)

- $\quad$ The Korean Guidelines

- $\quad$ The Asian Guidelines (2008)

- $\quad$ The HISORt Guidelines (Mayo clinic 2006)

- The International Consensus Diagnostic Criteria (ICDC) Guidelines (2011) [4].

In this discussion we shall focus on the ICDC guidelines that were formulated by a panel of experts at the $14^{\text {th }}$ Congress of the International Association of Pancreatology in 2011. The ICDC guidelines utilizes a combination of two or more of 5 cardinal features to make either a definitive or probable diagnosis of AIP. These cardinal features include:

- $\quad$ Radiological findings

- $\quad$ Serology

- $\quad$ Other organ involvement (OOI)

- Histology

- $\quad$ Response to steroids

Using type 1 AIP as the example, radiological imaging may reveal features typical of type 1 AIP such as diffuse enlargement with delayed enhancement or rim like enhancement. That constitutes level 1 evidence. Atypical features constitute level 2 evidence and may include segmental enlargement of delayed enhancement. If

Citation: Elizabeth Guelmo-Daisley., et al. "Pancreatic Pseudotumour in Seronegative Autoimmune Pancreatitis: A Rare case in the Caribbean". Acta Scientific Medical Sciences 6.2 (2022): 100-104. 
level 1 radiological evidence of AIP is found, then evaluation for OOI or serological evidence of AIP should be pursued. If either level 1 or 2 evidence for OOI or serology is present, then a definitive diagnosis of AIP may be made. However, if level 2 radiological evidence is present with level 2 evidence for OOI or serology or neither, then histology may be required to make the determination [4].

Due to the rarity of this disease entity in our setting, the tumefactive nature of the chronic pancreatitis, the lack of extra pancreatic manifestations of the disease and the deceptively normal IgG levels, our patient underwent management which superseded that which was required. The diagnosis was only made post operatively when histological evidence was available. The patient was then started on a course of corticosteroids to which she was readily responsive.

In 2008, Tassi., et al. found the immunological environment of pancreatic adenocarcinoma to be Thelper 2 (TH2) cell dominated. They discovered that these conditions impaired the body's natural anti- tumour immunological surveillance system namely, the CD4+ $\mathrm{T}$ cell response against the carcinoembryonic antigen CEA. The CD8+ T cell response to tumorigenesis was also suppressed. The pathogenesis of type 1 AIP is distinguished by a predominantly TH2 cell mediated response which generates increased serum IgG4 and IgG4 switched lymphocytes and plasma cells [5]. Thus theoretically, the immunological environment found in the setting of type 1 AIP is the ideal catalyst for pancreatic carcinogenesis. There have been numerous studies attempting to definitively prove that there is a cause-and-effect relationship between the two disease entities. However, due to relatively small sample sizes, short follow up periods, the lack of appropriate control groups and the retrospective design of most of these studies, the association between pancreatic adenocarcinoma and type 1 AIP has not been definitively proven $[6]$.

AIP and pancreatic carcinoma can coexist. Macinga., et al. in 2017 have shown this to be possible. A retrospective analysis of 295 pancreatic specimen resected for a focal mass lesion over a 14year period, revealed 6 individuals with both AIP and pancreatic ca [7]. The clinical implication of having synchronous disease entities means a pre-operative diagnosis of AIP does not eliminate the possibility of pancreatic ca, but introduces further dilemma into diagnosis and treatment of the disease.

\section{Conclusion}

Despite strict adherence to current clinical guideline, it is quite possible to confuse AIP with pancreatic malignancy. AIP is a rare disease entity in the Caribbean, the prevalence of which is unknown.

\section{Declaration}

The authors of this paper would like to declare that permission for the use of clinical information and images were obtained from the patient in the form of written consent. The patient is aware that all identifying information will be withheld to maintain anonymity.

\section{Conflicts of Interest}

There were no conflicts of interest.

\section{Financial Support and Sponsorship}

Nil.

\section{Bibliography}

1. de Castro SM., et al. "Incidence and characteristics of chronic and lymphoplasmacytic sclerosing pancreatitis in patients scheduled to undergo a pancreatoduodenectomy". HPB (Oxford) 12.1 (2010): 15-21.

2. Khandelwal A., et al. "Autoimmune Pancreatitis: an update". Abdominal Radiology 45.5 (2020): 1359-1370.

3. Masamune A., et al. "Nationwide epidemiological survey of autoimmune pancreatitis in Japan in 2016". Journal of Gastroenterology 55.4 (2020): 462-470.

4. Madhani K and Farrell JJ. "Autoimmune Pancreatitis: An Update on Diagnosis and Management". Gastroenterology Clinics of North America 45.1 (2016): 29-43.

5. Tassi E., et al. "Carcinoembryonic antigen - specific but not antiviral CD4+ T cell immunity is impaired in Pancreatic carcinoma patients". Journal of Immunology 181.9 (2008): 65956603.

6. Poddighe D. "Autoimmune pancreatitis and pancreatic cancer: Epidemiological aspects and immunological considerations". World Journal of Gastroenterology 27.25 (2021): 3825-3836.

7. Macinga P., et al. "Simultaneous occurrence of autoimmune pancreatitis and pancreatic cancer in patients resected for focal pancreatic mass". World Journal of Gastroenterology 23.12 (2017): 2185- 2193.

Citation: Elizabeth Guelmo-Daisley., et al. "Pancreatic Pseudotumour in Seronegative Autoimmune Pancreatitis: A Rare case in the Caribbean". Acta Scientific Medical Sciences 6.2 (2022): 100-104. 


\section{Assets from publication with us}

- Prompt Acknowledgement after receiving the article

- Thorough Double blinded peer review

- Rapid Publication

- Issue of Publication Certificate

- High visibility of your Published work

Website: www.actascientific.com/

Submit Article: www.actascientific.com/submission.php

Email us: editor@actascientific.com

Contact us: +919182824667 